Journal of Mathematics and Statistics 8 (2): 311-322, 2012

ISSN 1549-3644

(C) 2012 Science Publications

\title{
On Nonlinear Age- and Stage-Structured Population Models
}

\author{
Arild Wikan \\ Department of Business Administration and Social Sciences, \\ Harstad University College, Havnegata 5, N-9480 Harstad, Norway
}

\begin{abstract}
The scope of this review article is to present and discuss various aspects of discrete nonlinear age- and stage-structured population models. We show that such models cover species which exhibit a wide range of different life histories and that one may use them in order to deduce fairly general ecological principles with respect to stability and dynamical outcomes. From a mathematical point of view, we give several examples of the fact that the nonstationary dynamics generated by such maps is indeed rich as a result of different types of bifurcations of various nature as well as other mechanisms like frequency locking and crises that may occur.
\end{abstract}

Key words: Model formulation, nonstationary population dynamics, ecological implication

\section{INTRODUCTION}

In an influential review article "Simple mathematical models with very complicated dynamics" Sir May (1976) presented an analysis of a first order nonlinear difference equation often referred to as the logistic equation or the quadratic map. Both in May (1976) as well as in Sarkovskii (1964); Li and Yorke (1975); May and Oster (1976); Guckenheimer et al. (1977); Feigenbaum (1978) and Singer (1978) it was demonstrated that simple one-dimensional nonlinear maps of biological relevance could exhibit an extraordinary rich dynamical behaviour ranging from stable fixed points, periodic points to chaotic behaviour. An excellent summary of results, in particular results from the quadratic map, may be obtained in Thunberg (2001), see also Jost (2005).

However, from a biological point of view there is a variety of cases where one-dimensional population models are not sufficient modelling tools. A more realistic approach is to apply age-structured models. Such matrix models (often referred to as Leslie matrix models) were independently developed in the 1940's by Bernardelli (1941); Lewis (1942) and Leslie (1945; 1948) but perhaps somewhat strange not widely adopted by ecologists until the 1970's. Originally, the models were linear and various dynamical outcomes may be found in Cull and Vogt $(1973 ; 1974)$ and Hallam and Levin (1986), Later, density dependent or nonlinear terms were considered too, cf. the classical papers by Guckenheimer et al. (1977) and the striped bass fishery model by Levin and Goodyear (1980). As accounted for by Caswell (2001), state variables others than age (for example size) were introduced in population models by Patten (1976); Boling (1973); see also Metz and Diekmann (1986); Hallam et al. (1990) and Gurney et al. (1990). Such models are called stagestructured models and have been applied on several different species as well as being analysed from theoretical perspectives. Cushing (1998) and Caswell (2001) the analysis of a wide range of stage-structured matrix models is included.

In this review study focus is mainly on three different topics. First we will discuss the models and the prerequisites they rest upon and together with lots of references show how the models may and have been applied to a large number of cases of ecological relevance. In another direction focus is on possible nonstationary and chaotic dynamics. Avoiding mathematical details and proofs we present several examples involving bifurcations of different type and nature as well as other mechanisms like frequency locking and crisis and show that the dynamics found in these multidimensional maps is much richer than we may find in one-dimensional cases. Finally we discuss ecological implications. In particular we address the question whether it is possible to formulate some general ecological principles with respect to stability and nonstationary behaviour of populations who possess different life histories.

Models: We start by presenting the age-structured model. At time $t$ we split the population $x_{t}$ into $n$ distinct nonoverlapping age classes $x_{t}=\left(x_{1, t}, \ldots, x_{n, t}\right)^{T}$ where the total population $\mathrm{x}$ is given by $\mathrm{x}=\mathrm{x}_{1}+\cdots+$ $x_{n}$. The relation between $x$ at two consecutive time steps may be expressed as a map Eq. 1: 
$\mathrm{x} \rightarrow \mathrm{Ax}$

where, the Leslie matrix $\mathrm{A}$ is on the form Eq. 2:

$$
\mathrm{A}=\left(\begin{array}{llll}
\mathrm{f}_{1} & \mathrm{f}_{2} & \cdots & \mathrm{f}_{\mathrm{n}} \\
\mathrm{p}_{1} & 0 & \cdots & 0 \\
0 & & & \\
\vdots & \ddots & & \\
0 & & \mathrm{p}_{\mathrm{n}-1} & 0
\end{array}\right)
$$

Moreover, $\mathrm{f}_{\mathrm{i}}$ is the average fecundity (number of daughters born per female) of a member of the $i^{\text {th }}$ age class at time $t$ and $p_{i}$ may be interpreted as the (year to year) survival probability. In maps like (1) there is an implicit assumption that sexual maturity is linked to age or that other properties than age are irrelevant. Another possibility is that if such relevant properties exist they must be highly correlated with age.

The matrix elements may be nonlinear (density dependent) or not. Considering two age classes where members of the oldest age class prey upon members of the youngest class (cannibalism) a natural assumption could be $\mathrm{p}=\mathrm{p}\left(\mathrm{x}_{2}\right)$. In fishery models it is often assumed that older individuals contribute more than younger individuals to density effects, therefore one may suppose (Levin and Goodyear, 1980) $\mathrm{f}=\mathrm{f}(\mathrm{y})$ where $\mathrm{y}=$ $\alpha_{1} x_{1}+\cdots+\alpha_{n} x_{n}$ (a weighted sum of age classes). A third possibility is that $\mathrm{f}$ (or $\mathrm{p}$ ) depends on the total population $\mathrm{x}$. This is a natural choice in this study since one of our main goals is to compare dynamics of species who possess very different life histories.

Frequently used density functions are members of the Deriso-Schnute family $\mathrm{f}_{\mathrm{i}}=\mathrm{F}_{\mathrm{i}}(1-\gamma \beta \mathrm{x})^{1 / \gamma}, \beta>0$ (Bergh and Getz,1988; Tuljapurkar et al., 1994) but see Getz (1996) for other examples. Thus, we write the fecundity (or and the survival) as a product of a constant term $\mathrm{F}_{\mathrm{i}}$ and a density dependent term (1 $\gamma \beta \mathrm{x})^{1 / \gamma}$. If $\gamma=-1$ we have the well-known compensatory Beverton and Holt relation, if $\gamma \rightarrow 0$ then we arrive at $f_{i}$ $=F_{i} \exp (-\beta x)$ (or $\left.p_{i}=P_{i} \exp (-\beta x)\right)$ which is called the overcompensatory Ricker relation inspired by the seminal study by Ricker (1954). In this review we shall deal exclusively with the Ricker case.

The dynamics of a large number of ecological populations has been modelled by (1). Linear agestructured models (constant fecundities and constant survivals) have for example been applied on rabbits (Darwin and Williams, 1964), great tits (Pennycuick, 1969) and trout (Beland, 1974). In case of nonlinear models we refer to Cooke and Leon (1976) (birds), Longstaff (1977) (beetles), Levin and Gooodyear (1980) (striped bass), see also Hastings (1984);
Desharnais and Liu (1987); Burkey and Stenseth (1994) and Nisbet and Onyiah (1994). References to lots of other examples may be found in Caswell (2001). Guckenheimer et al. (1977); Silva and Hallam (1993) and Wikan and Mjolhus (1995; 1996) have paid attention to the dynamics of (1) in unstable and chaotic parameter regions. Wikan (1997); Davydova et al. (2003); Mjolhus et al. (2005) and Cushing (2006) focus is on the dynamics of species who reproduce only once at the end of their lifes. Ergodic results may be obtained in Cushing (1988; 1989) and Crowe (1994).

Unlike (1), in stage-structured models we do not divide the population into nonoverlapping age classes. Instead, we split the population into stages, for example one sexual immature stage and one sexual mature stage. The motivation for doing so is that there may be other factors which are more important with respect to maturity than age. As already mentioned, one such factor is body size. Indeed, following Caswell (2001) "size dependent demography is probably the rule rather than the exception". Examples which link body size to sexual maturity may be found among plants (Klinkhamer et al., 1987a; 1987b), crabs (Campbell and Eaglis, 1983; Botsford, 1986; 1992). See also lots of references in Caswell (2001). Among several alternatives we will in this study consider the two-stage model, first proposed by Neubert and Caswell (2000):

$$
\mathrm{x} \rightarrow \mathrm{Bx}
$$

where, the transition matrix B is on the form:

$$
\mathrm{B}=\left(\begin{array}{cc}
\mu_{1}(1-\mathrm{p}) & \mathrm{f} \\
\mu_{1} \mathrm{p} & \mu_{2}
\end{array}\right)
$$

The meaning of the entries in (4) is as follows: $\mu_{1}$ and $\mu_{2}$ are the fractions of the immature population $\mathrm{x}_{1}$ and the mature (adult) population $\mathrm{x}_{2}$ respectively, which survive from time $\mathrm{t}$ to $\mathrm{t}+1 . \mathrm{x}=\mathrm{x}_{1}+\mathrm{x}_{2}$ is the total population. $\mathrm{p}$ is the fraction of the immature population which survives to become adult and $f$ is the fecundity. Just as in (2) the elements of (4) may be nonlinear or not. Neubert and Caswell (2000) consider several submodels. In one submodel it is assumed that $\mu_{1}=\mu_{1}$ $\exp (-\mathrm{x})$ while the other parameters are constant. In another, $\mathrm{p}=\mathrm{p} \exp (-\mathrm{x})$ is the only nonlinear term and so on. One some occasions it may be convenient to apply three-stage models. Indeed, in the celebrated collembolan study, Cushing et al. (1996); Costantino et al. (1997) and Dennis et al. (1997) divided the population into three stages, larvae $\mathrm{L}$, pupae $\mathrm{P}$ and adult insects $\mathrm{A}$ and in fact showed that their LPA model could not only describe but even predict chaotic 


\section{J. Math. \& Stat., 8 (2): 311-322, 2012}

behaviour in laboratory insect populations. More on this model, model as well as properties of other stagestructured models may be found in Cushing (1998) and regarding permanence, cf. Kon et al. (2004).

Although (1) and (3) rest on different prerequisites both of them cover species with several life histories. Indeed, if $F_{i}=0, i<n$ and $F_{n}>0$ in (2) or $\mu_{2} \rightarrow 0$ in (4) the population is semelparous (i.e., reproducing only once). If several $\mathrm{F}_{\mathrm{i}}>0$ or $\mu_{2} \rightarrow 1$ the population is iteroparous (repeated reproduction). The subclass $\mathrm{F}_{1}=$ $0, \mathrm{~F}_{2}>0$ (two age classes) or $\mu_{2} \rightarrow 0, \mathrm{p} \rightarrow 1$ is often referred to as precocious semelparity which covers species with rapid development followed by only one reproduction, for example biennials and annual plants. Delayed semelparity occurs when $n$ is larger $F_{i}>0, i<$ $\mathrm{n}, \mathrm{F}_{\mathrm{n}}>0$ or $\mu_{2} \rightarrow 0$ and $0<\mathrm{p}<1$. Typical examples are periodical cicadas, Behncke (2000) and Davydova et al. (2003) and several salmon species that live for many years before they become mature and reproduce only once. We may also divide the iteroparous case into two subclasses. The subclass $\mathrm{F}_{\mathrm{i}}>0, \mathrm{i} \leq \mathrm{n}\left(\mathrm{F}_{1}=0, \mathrm{~F}_{\mathrm{i}}>0,2 \leq\right.$ $\mathrm{i} \leq \mathrm{n})$ or $\mu_{2} \rightarrow 1, \mathrm{p} \rightarrow 1$ is classified as precocious iteroparity and covers several small mammals species (small rodent species) which start to reproduce at young age and may survive to reproduce for several years. The fourth subclass, delayed iteroparity, is characterized by several nonfertile age classes followed by several age classes who are fertile, for example $F_{i}=0, i \leq n / 2, F_{i}>$ $0, \mathrm{n} / 2<\mathrm{i} \leq \mathrm{n}$ or $0<\mu_{2}<1,0<\mathrm{p}<1$. In this subclass we find humans and other large mammals.

Finally, we will also like to mention some other model strategies. Difference delay equation models are models on the form $\mathrm{x}_{\mathrm{t}+1}=\mathrm{g}\left(\mathrm{x}_{\mathrm{t}}, \mathrm{x}_{\mathrm{t}-\mathrm{T}}\right)$ where $x$ is the size of the population and $\mathrm{T}$ the time from birth to maturity. Such models may be regarded as aggregated versions of (1) where detailed information of the dynamics within age classes is neglected, cf. the Baleen model by Clark (1976). In case of other species we refer to Botsford (1986; 1992); Tuljapurkar et al. (1994) and Higgins et al. (1997). One may also incorporate spatial structure in matrix models, see for example Gyllenberg et al. (1993). In wildlife protection studies one often divides the habitat of a species in a reserve and a harvest zone. Regarding fish populations several interesting properties of such a strategy is discussed in Hastings and Botsford (1999). There are also discrete time growth dispersal models, i.e., models where growth is modelled in discrete time while dispersal is modelled in continuous time. Kot and Schaffer (1986); Neubert et al. (1995) and Lutscher and Lewis (2004) provide excellent examples.

Analysis and examples: Under the assumption that all density dependent terms are on Ricker form and functions of the total population both (1) and (3) possess a unique nontrivial fixed point. In (1) we express the fixed point as $\left(\mathrm{x}_{1}{ }^{*}, \ldots, \mathrm{x}_{\mathrm{n}}{ }^{*}\right)$ where $\mathrm{x}^{*}=\mathrm{x}_{1}{ }^{*}+$ $\cdots+\mathrm{x}_{\mathrm{n}}{ }^{*}$ is the total equilibrium population. In (3) we denote the fixed point as $\left(\mathrm{x}_{1}{ }^{*}, \mathrm{x}_{2}{ }^{*}\right)$. Stability analysis is performed by linearizing about the fixed point which in turn gives birth to an $n$th degree eigenvalue equation. Provided all eigenvalues $\lambda$ are located in the inside of the unit circle in the complex plane the fixed point is stable. If we increase a parameter (in this study we will restrict the analysis to an increase of the fecundity) such that an eigenvalue leaves the unit circle the fixed point becomes unstable. The parameter value at which this takes place may be termed the threshold. As is wellknown, the location where an eigenvalue leaves the unit circle has crucial impact on the nonstationary dynamics at parameter values just beyond threshold. Just as in one-dimensional maps an eigenvalue may leave through $\lambda=1$ or $\lambda=-1$. If $\lambda=1$, the general case is that the fixed point will undergo a saddle node bifurcation at threshold. The other possibilities are the pitchfork and the transcritical bifurcations. $\lambda=-1$ results in a flip (period doubling) bifurcation. The third possibility is that a pair of modulus 1 complex valued eigenvalues leave the unit circle at threshold. Then the fixed point will go through a Hopf (Neimark Sacker) bifurcation. A detailed description of all bifurcations referred to above may be obtained in Devaney (2003) or Guckenheimer and Holmes (1983). We shall now provide several examples where focus is on the nonstationary dynamics.

Example 1 (Precocious iteroparity): Assuming constant fecundities (indicated by capital letters $\mathrm{F}_{\mathrm{i}}$ ) $\mathrm{F}_{1}=$ $0, \mathrm{~F}_{2}=\ldots=\mathrm{F}_{\mathrm{n}}>0$ and density dependent survivals $\mathrm{p}_{\mathrm{i}}=$ $\mathrm{P}_{\mathrm{i}} \exp (-\alpha \mathrm{x}), \alpha>0,0<\mathrm{P}_{\mathrm{i}} \leq 1$ we may express (1) as:

$\left(\mathrm{x}_{1}, \ldots, \mathrm{x}_{\mathrm{n}}\right) \rightarrow\left(\mathrm{F}_{2} \mathrm{x}_{2}+\cdots+\mathrm{F}_{\mathrm{n}} \mathrm{x}_{\mathrm{n}}, \mathrm{p}_{1} \mathrm{x}_{1}, \mathrm{p}_{2} \mathrm{x}_{2}, \ldots, \mathrm{p}_{\mathrm{n}-1} \mathrm{x}_{\mathrm{n}-1}\right)$

Now, using the fact that $F$ is large at instability an asymptotic argument (Wikan and Mjolhus, 1995; Wikan, 1998) shows that the solution of the $n$th order eigenvalue equation (at instability) is very close to the solution of the eigenvalue equation when $n=2$. This has the immediate consequence that the dynamics of the $n$ age class model (5) is maintained already in the two age class case. Hence, in (5), it suffices to consider $\mathrm{n}=2$. Then $\mathrm{x}^{*}=\alpha^{-1} \ln \left(\mathrm{FP}_{1}\right)$ and whenever $\mathrm{F}$ is small we find from the Jury criteria (Murray, 2003) that the fixed point $\left(\mathrm{x}_{1}{ }^{*}, \mathrm{x}_{2}{ }^{*}\right)$ is stable. By increasing $\mathrm{F}$ we also increase $x^{*}$ and when $\mathrm{F}=\mathrm{F}_{\mathrm{H}}$ where $\mathrm{F}_{\mathrm{H}}$ is defined through $\mathrm{F}_{\mathrm{H}}{ }^{-1} \exp \left[2\left(1+\mathrm{F}_{\mathrm{H}}\right) / \mathrm{F}_{\mathrm{H}}\right]=\mathrm{P}_{1}$ the fixed point undergoes a supercritical Hopf bifurcation. 


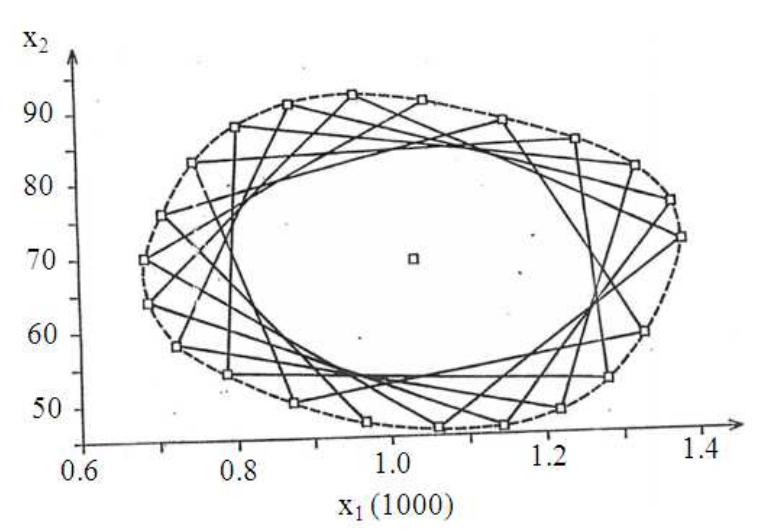

(a)

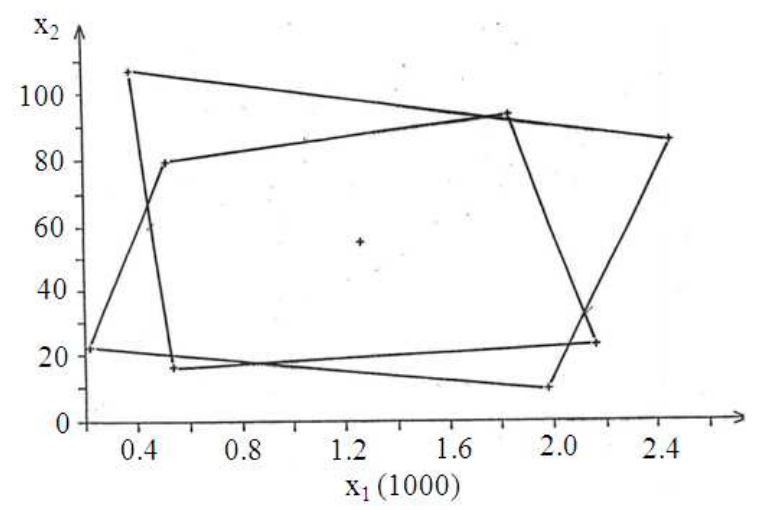

(c)

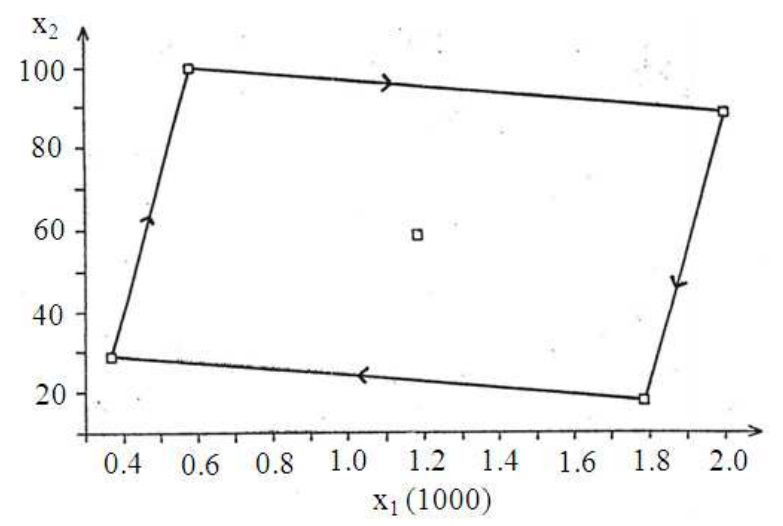

(b)

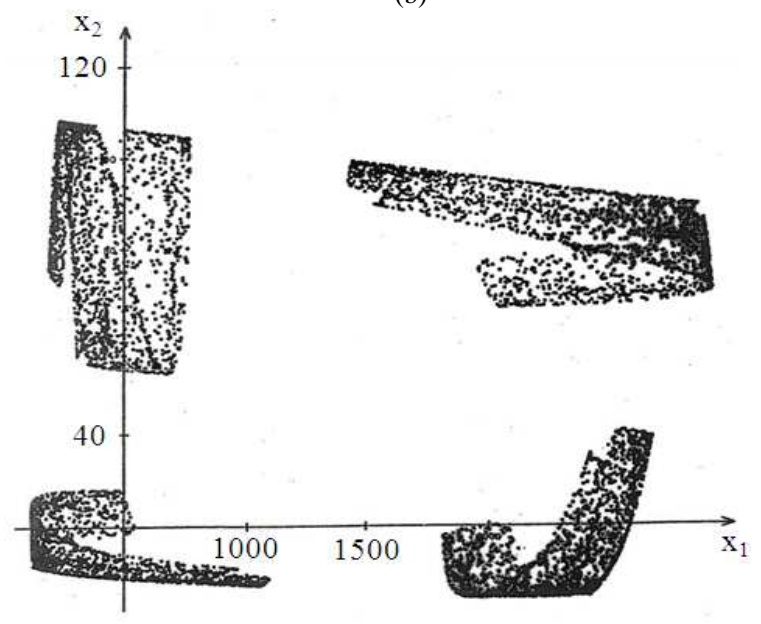

(d)

Fig. 1: (a) A quasistationary (almost 4 periodic) orbit (solid line) restricted to an invariant curve (dashed line). Parameter values $\alpha=0.02, \mathrm{P}=0.6$ and $\mathrm{F}=15$. Only the 20 last iterations are shown; (b) An exact 4 -periodic orbit generated by map (5). Parameter values as in Fig. 1a but $\mathrm{F}=20$; (c) An exact 8-periodic orbit. $\mathrm{F}=22$; (d) Map (5) in the chaotic regime. $\mathrm{F}=27$. The attractor has been divided into 4 disjoint subsets which are visited once every fourth iteration

Thus, if $\mathrm{F}>\mathrm{F}_{\mathrm{H}}$ and $\left|\mathrm{F}-\mathrm{F}_{\mathrm{H}}\right|$ is small the dynamics is restricted to an attracting invariant curve which surrounds the unstable fixed point as displayed in Fig. 1a. On such a curve (5) is topological equivalent to a circle map which means that (5) does nothing but move or rotate points around the curve. Associated with a circle map there is also a rotation number $\sigma$ which in this context (see Guckenheimer and Holmes (1983) can be expressed as $\sigma=\mathrm{c}+(\mathrm{bd} / \mathrm{a})\left(\mathrm{F}_{\mathrm{H}^{-}} \mathrm{F}\right)$ where $\mathrm{c}=\arg \lambda$. If $\sigma$ is irrational an orbit of a point is often referred to as a quasistationary orbit. If $\sigma=1 / \mathrm{n}$ rational, the dynamic outcome is an orbit of period n. Also, note that whenever $\sigma$ is rational for a given parameter value $\mathrm{F}=$ $\mathrm{F}_{\mathrm{r}}$ it follows from the implicit function theorem that there exists an open interval about $F_{r}$ where the periodicity is maintained. This phenomenon is known as frequency locking of periodic orbits.
Now, returning to our example, $\lambda$ is close to the imaginary axis at bifurcation threshold (i.e., $\mathrm{c}=\arg \lambda \approx$ $\pi / 2$. Consequently, close to the bifurcation $\sigma \approx 1 / 4$ which is clearly seen in Fig. 1a and if we continue to increase $\mathrm{F}$ an exact 4-period orbit (frequency locking) is established. This is shown in Fig. 1b. When F becomes even larger we find that the fourth iterate of (5) undergoes a flip bifurcation which results in an 8-period orbit which is illustrated in Fig. 1c. Through further enlargement of $F$ new flip bifurcations occur which result in orbits of period 16, 32 and 64 respectively. From a mathematical point of view the dynamics in this part of parameter space is similar to what one finds in onedimensional maps, see the review papers by May (1976) and Thunberg (2001). Eventually the dynamics becomes chaotic as displayed in Fig. 1d. Note that the attractor is divided in 4 disjoint subsets and that each of the subsets 
are visited once every fourth iteration so there is a kind of 4 -periodicity preserved even in the chaotic regime. In Fig. 2 we show a "4-period" chaotic attractor generated by (5) in case of $n=3$. A detailed analysis of (5) may be obtained in Wikan and Mjolhus (1995).

Example 2 (Delayed semelparity): A slightly different situation occurs in the 3-age class map:

$\left(\mathrm{x}_{1}, \mathrm{x}_{2}, \mathrm{x}_{3}\right) \rightarrow\left(\mathrm{Fx}_{3}, \mathrm{px}_{1}, \mathrm{px}_{2}\right)$

where, $p=P \exp (-x)$. In case of small and "moderate" $F$ values the dynamics of (6) is similar to what we found from (5). Whenever $\mathrm{F}$ is small, $\left(\mathrm{x}_{1}{ }^{*}, \mathrm{x}_{2}{ }^{*}, \mathrm{x}_{3}{ }^{*}\right)$ is stable and when we increase $F$ to a level $F_{H}$ a supercritical Hopf bifurcation occurs. Moreover, $\sigma \approx 1 / 4$ at threshold and here too we experience the existence of a fecundity interval beyond $\mathrm{F}_{\mathrm{H}}$ where the dynamics is exact 4-periodic.

However, if we continue to increase $F$ the result is not a flip bifurcation which creates an exact 8-period orbit. Instead we find that the fourth iterate of (6) undergoes a supercritical Hopf bifurcation at a threshold $\mathrm{F}=\mathrm{F}_{\mathrm{H} 2}$. Therefore, beyond $\mathrm{F}_{\mathrm{H} 2}$ and in case if $\left|\mathrm{F}-\mathrm{F}_{\mathrm{H} 2}\right|$ small the dynamics is restricted to 4 disjoint invariant attracting curves which are visited once every fourth iteration. This is displayed in Fig. 3a. As we continue to increase $\mathrm{F}$ the curves become twisted which signal that we are on the onset to chaos. In Fig. $3 \mathrm{~b}$ we show the chaotic attractor in case of $\mathrm{F}=55$.

Example 3 (Precocious iteroparity): Next, consider:

$$
\left(\mathrm{x}_{1}, \ldots, \mathrm{x}_{\mathrm{n}}\right) \rightarrow\left(\mathrm{fx}_{1}+\cdots+\mathrm{fx}_{\mathrm{n}}, \mathrm{Px}_{1}, \mathrm{Px}_{2}, \ldots, \mathrm{Px}_{\mathrm{n}-1}\right)
$$

where, $f=F \exp (-x)$ and constant year-to-year survival probabilities $\mathrm{P}, 0<\mathrm{P} \leq 1$. The unique nontrivial fixed point is:

$\left(x_{1}^{*}, x_{2}^{*}, \ldots, x_{n}^{*}\right)=\left(\frac{1}{K} x^{*} \cdot \frac{P}{K} x^{*}, \ldots, \frac{P^{n-1}}{K} x^{*}\right)$

$\mathrm{K}=\sum_{\mathrm{i}=0}^{\mathrm{n}-1} \mathrm{P}^{\mathrm{i}}$ and $\mathrm{x}^{*}=\ln (\mathrm{FK})$ and the eigenvalue equation may be cast in the form:

$\lambda^{\mathrm{n}}-\frac{\left(1-\mathrm{x}^{*}\right)}{\mathrm{K}} \sum_{\mathrm{i}=0}^{\mathrm{n}-1} \mathrm{P}^{\mathrm{i}} \lambda^{\mathrm{n}-1-\mathrm{i}}=0$

Here, we concentrate on the case $\mathrm{n}=2$. Then from (9) and the Jury criteria we find that $\left(\mathrm{x}_{1}{ }^{*}, \mathrm{x}_{2}{ }^{*}\right)$ is stable in case of $\mathrm{F}$ small and will undergo supercritical bifurcations at the thresholds:

$$
\mathrm{F}=\left\{\mathrm{F}_{\mathrm{F}}=(1 /(1+\mathrm{P})) \exp [2 /(1-\mathrm{P})] \quad 0<\mathrm{P}<1 / 2 \quad\right. \text { (10a) }
$$

$$
\mathrm{F}=\left\{\mathrm{F}_{\mathrm{H}}=(1 /(1+\mathrm{P})) \exp [(1+2 \mathrm{P}) / \mathrm{P}] \quad 1 / 2<\mathrm{P}<1\right.
$$

where, the subscripts $\mathrm{F}$ and $\mathrm{H}$ refer to a flip and a Hopf bifurcation respectively. From a mathematical point of view the most interesting interval is $1 / 2<\mathrm{P}<1$ and in the bifurcation diagram, Fig. 4, we have summarized the result when $P=0.9$. For small values of $F$ there is one stable attractor, namely the fixed point $\left(\mathrm{x}_{1}{ }^{*}, \mathrm{x}_{2}{ }^{*}\right)$. When $F$ is increased to 10.036 the third iterate of (7) undergoes a saddle node bifurcation which gives birth to a stable 3-cycle but also an unstable 3-cycle (not visible in the diagram). Thus, if $10.036<\mathrm{F}<12.20$ there are two stable attractors and the ultimate fate of an orbit depends on the initial condition. At the threshold $F$ $=11.81$ (cf. (10b)) the fixed point undergoes a supercritical Hopf bifurcation and an attracting invariant curve is established. Due to the fact that $\lambda$ is close to third root of unity at bifurcation there is an almost 3-period orbit restricted to the curve. Hence, in the interval 11.81 $<\mathrm{F}<12.20$ a 3-cycle of large amplitude coexists with an almost 3-cyclic attractor of small amplitude. At $\mathrm{F}=$ 12.20 the invariant curve is hit by the unstable 3-cycle created at the earlier value 10.036. This makes the invariant curve disappear, hence when $\mathrm{F}>12.20$ there is only one attractor, the 3-cycle. At even higher $\mathrm{F}$ values successive flip bifurcations take place generating orbits of period $3 \cdot 2^{\mathrm{k}}, \mathrm{k}=1,2, \ldots$ Eventually the dynamics becomes chaotic. For details and further analysis of (7), cf. Wikan and Mjolhus (1996).

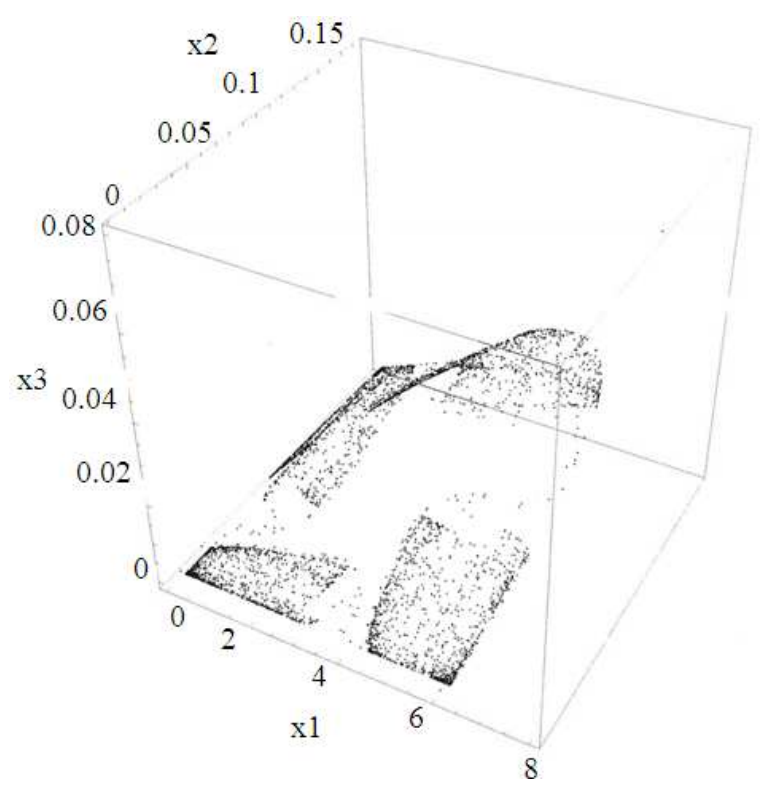

Fig. 2: A chaotic attractor generated by (5) in case of 3 age classes 


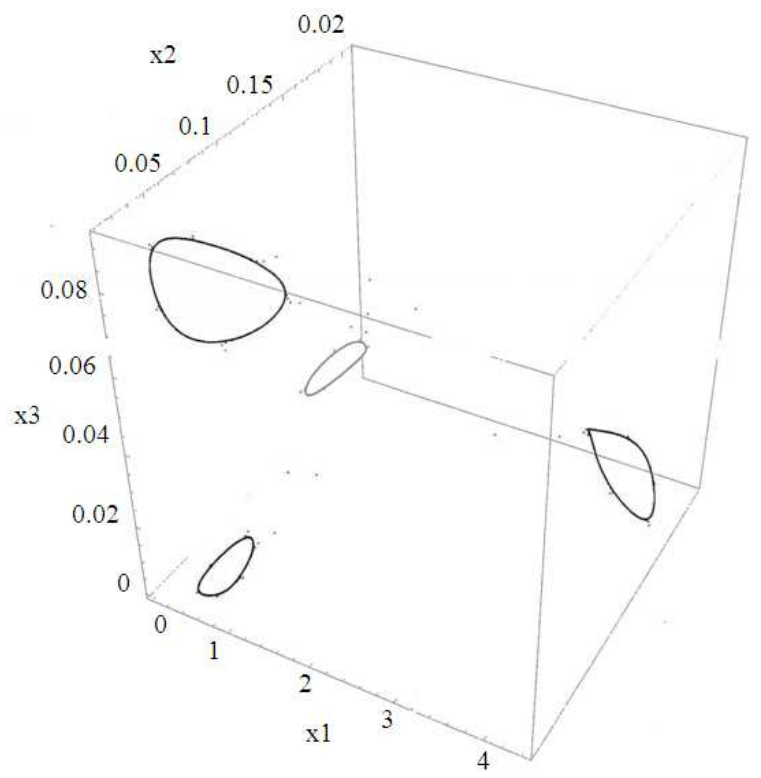

(a)

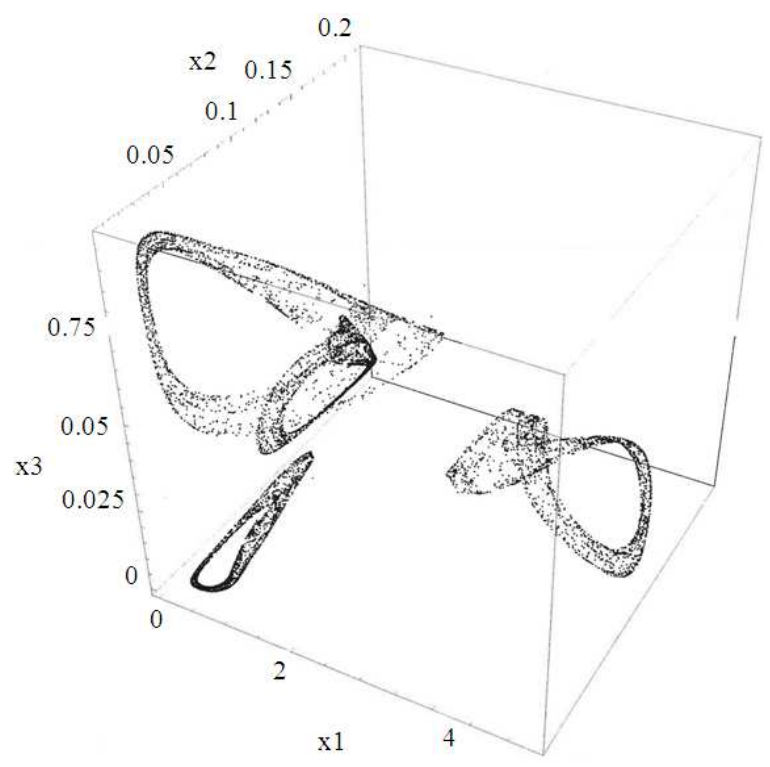

(b)

Fig. 3: (a) Map (6) after the secondary Hopf bifurcation $(\mathrm{P}, \mathrm{F})=(0.6,48)$; (b) Map (6) in the chaotic regime $(\mathrm{P}, \mathrm{F})=(0.6,55)$

As we have seen bifurcations can be of both supercritical and subcritical nature. If a fixed point undergoes a supercritical bifurcation when the parameter $\mu$ is increased to a value $\mu_{0}$ there is established an attracting orbit (stable 2-cycle in the flip case, a quasiperiodic orbit restricted to an invariant attracting curve in the Hopf case) above $\mu_{0}$. In the subcritical case such attractors do not exist. Now, if we shall prove that a Hopf bifurcation which occurs in a two-dimensional map is super- or subcritical we first write the map on complex form and provided $\lambda^{3} \neq 1, \lambda^{4}$ $\neq 1$ (that means we are outside the strong resonance cases) we may through a series of near identity transformations (normal form calculations) express it as (Govaerts and Ghaziani, 2006):

$\mathrm{z} \rightarrow \mathrm{ze}^{\mathrm{i} \theta_{0}}\left(1+\mu+\mathrm{d}|\mathrm{z}|^{2}\right)+\mathrm{O}\left(|\mathrm{z}|^{4}\right)$

and the sign of $a=\operatorname{Re}(d)$ will determine the nature of bifurcation (a $<0$ implies supercritical, $a>0$ subcritical). a is a complicated formulae which first was established by Wan (1978). Wikan (1998) it is proved that the fixed point of a map like the one considered in Example 1 where the density dependent survivals belong to the Deriso-Schnute family undergoes a supercritical Hopf bifurcation at instability. The nature of flip bifurcations in two-dimensional maps can be studied by first performing a center manifold restriction and then express the map as:

$\mathrm{w} \rightarrow-(1+\mu) \mathrm{w}+\mathrm{aw}^{3}+\mathrm{O}\left(\mathrm{w}^{4}\right)$

where, $w$ is real. Details and stability formulaes may be found in Guckenheimer and Holmes (1983). Finally, it should also be mentioned that there exist several numerical packages designed for studying bifurcation phenomena, see for example Ueta et al. (1999); Kuznetsov (2004) and Kuznetsov and Meijer (2005). In particular, confer Govaerts and Ghaziani (2006) where they use the MATLAB package CL_MatCont on bifurcation problems in a stage-structured cannibalism model. Our experience is that supercritical bifurcations dominate in age and stage-structured models like (1) and (3).

Next, let us briefly focus on possible routes to chaos. Referring to Example 1 we have the route: Stable fixed point $\rightarrow$ Invariant curve, quasiperiodic orbit (Hopf) $\rightarrow$ Periodic orbit (frequency locking) $\rightarrow$ Periodic orbits where the periods are doubled (successive flip bifurcations) $\rightarrow$ chaos. Hence, once a periodic orbit is established the route to chaos is similar to what one finds in one-dimensional maps as summarized in Thunberg (2001). Another possibility is that once a periodic orbit is established there may be a secondary Hopf bifurcation and chaotic behaviour may evolve when the disjoint subsets start to get twisted, cf. Example 2 and Fig. 3b. A third scenario is that one from the periodic orbit may return to quasiperiodicity again as the bifurcation parameter increases. It is also possible to go directly from quasiperiodicity (invariant curve) to chaos. This may happen as the invariant curve starts to break up. An example may be found in Wikan (2012) by use of the Neubert and Caswell map (3) 
(density dependent $\mu_{1}$ values). We may also face the situation that the invariant curve becomes kinked so that it is not topologically equivalent to a circle anymore (see Wikan (1998) by use of small positive $\gamma$ values in the Deriso-Schnute model). A comprehensive study of crises (collisions of attractors and unstable periodic orbits) in Leslie matrix models may be obtained in Ugarcovici and Weiss (2004) (see also Example 3). Crises as well as subcritical bifurcations may lead to large and discontinuous population changes.

Example 4 (Precocious and delayed semelparity): First we consider the precocious case where both fecundity and survival probability is density dependent, i.e.:

$$
\left(\mathrm{x}_{1}, \mathrm{x}_{2}\right) \rightarrow\left(\mathrm{fx}_{2}, \mathrm{px}_{1}\right)
$$

where, $f=F \exp (-\alpha x)$ and $p=P \exp (-\beta x)$. The model possesses a unique nontrivial fixed point $\left(\mathrm{x}_{1}{ }^{*}, \mathrm{x}_{2}{ }^{*}\right)$ and it is stable provided:

$$
\begin{aligned}
& (\beta-\alpha)\left[1-P \exp \left(-\beta \mathrm{x}^{*}\right)\right] \mathrm{x}_{1}^{*}>0 \\
& 2-\left(\beta \mathrm{x}_{1}^{*}+\alpha \mathrm{x}_{2}^{*}\right)>0
\end{aligned}
$$

(Wikan, 2012). Now, if $\beta>\alpha$ it is obvious that $\left(\mathrm{x}_{1}{ }^{*}, \mathrm{x}_{2}{ }^{*}\right)$ is stable in case of small equilibrium populations $\mathrm{x}^{*}$. Regarding the nonstationary dynamics, $\beta \gg \alpha$ gives the same qualitative picture as accounted for in Example 1, 3 -periodic dynamics is also a possibility. If $\beta>\alpha$ and $\beta$ $\alpha$ small we detect quasistationary orbits. Next, assume $\beta$ $\leq \alpha$. Then the left hand side of (14a) is always negative (or equal to zero if $\beta=\alpha$ ). Consequently, the nontrivial fixed point is always unstable. Since (14a) is associated with the possibility that $\left(\mathrm{x}_{1}{ }^{*}, \mathrm{x}_{2}{ }^{*}\right)$ shall undergo a flip bifurcation at instability threshold it is natural to seek for a stable 2-cycle in case of $x^{*}$ small. Indeed, such a stable 2-cycle exists and the points in the cycle are on the form $\left(\hat{\mathrm{x}}_{1}, \hat{\mathrm{x}}_{2}\right)=(\mathrm{A}, 0)$ or $(0, \mathrm{~B})$ which implies that only one age class is populated at each time. (If $\alpha=1$ and $\beta=0$ then $\mathrm{A}=\mathrm{P}^{-1} \ln (\mathrm{FP})$ and $\mathrm{B}=\ln (\mathrm{FP})$ ). Dynamics in which only one age class is populated at each time has been referred to as SYC (Single Year Class) dynamics, cf. Mjolhus et al. (2005). It has also been termed synchronization, see Bulmer (1977). As far as we know Bulmer (1977) appears to be the first to have noticed SYC dynamics in theoretical models, see also Wikan and Mjolhus (1996); Behncke (2000); Mjolhus et al. (2005) and Cushing (2006). Precise results of conditions which lead to SYC dynamics and conditions where age classes can coexist may also be obtained in a slightly different model analysed by Davydova et al. (2003). Turning to delayed semelparity we consider:

$$
\left(\mathrm{x}_{1}, \ldots, \mathrm{x}_{\mathrm{n}}\right) \rightarrow\left(\mathrm{fx}_{\mathrm{n}}, \mathrm{Px}_{1}, \ldots, \mathrm{Px}_{\mathrm{n}-1}\right)
$$

where, $\mathrm{f}=\mathrm{F} \exp (-\mathrm{x})$, see Mjolhus et al. (2005). The only dynamics we find in case of $\mathrm{x}^{*}=\ln \left(\mathrm{FP}^{\mathrm{n}-1}\right)$ small is an attracting n-cycle of SYC form:

$$
\left(\mathrm{P}^{1-\mathrm{n}} \mathrm{x}^{*}, 0, \ldots, 0\right), \ldots,\left(0, \ldots, 0, \mathrm{P}^{\mathrm{i}-\mathrm{n}} \mathrm{x}^{*}, 0, \ldots, 0\right) \ldots\left(0, \ldots, 0, \mathrm{x}^{*}\right)
$$

Assuming $\mathrm{n}$ even, as $\mathrm{x}^{*}$ becomes larger successive flip bifurcations create stable SYC cycles of period $2^{k} n$. Also in the chaotic regime we only find SYC dynamics. When $\mathrm{n}$ is odd much of the same picture emerges. The only difference is that in case of intermediate values of $\mathrm{x}^{*}$ there may exist small parameter windows where the fixed point is stable, cf. Mjolhus et al. (2005).

However, note that SYC dynamics is not a very likely outcome in stage-structured models, see the discussion in Kon (2005). Indeed, Neubert and Caswell (2000) present by use of (3) an interesting analysis of the precocious semelparous case $\sigma_{2}=0.1, \mathrm{p}=0.9$. They found a stable nontrivial equilibrium in case of $\mathrm{F}$ small and when $F$ was increased (they considered $F$ values as large as $10^{7}$ ) the dynamics alternated between stable orbits of low period and chaotic regions in the sense that prior to a chaotic region the period of an orbit was $\mathrm{q}$ and after the region the period was found to be $\mathrm{q}+1$. This phenomenon is known as period adding cycles and may also be found in the age-structured model by Guckenheimer et al. (1977). (We urge the reader to extend the bifurcation diagram in Fig. 4 and see if the same happens there too).

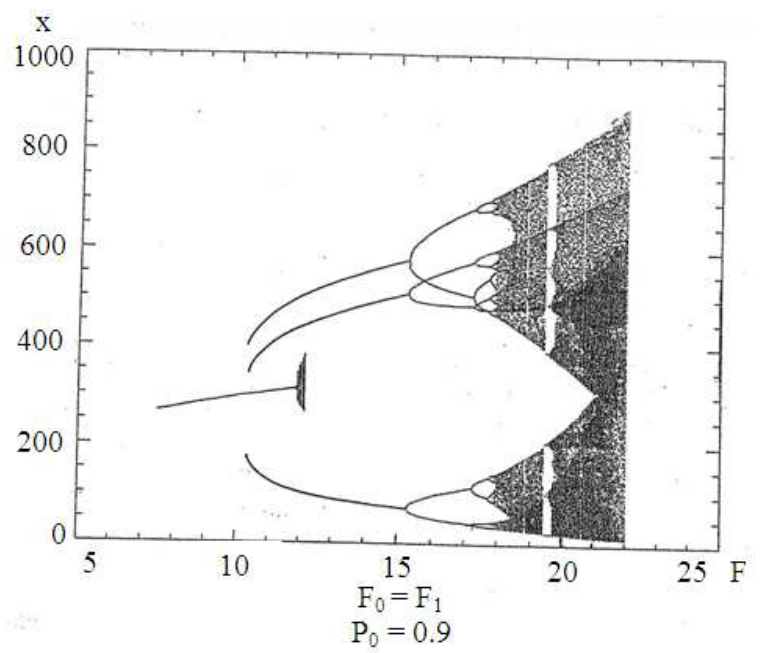

Fig. 4: Bifurcation diagram generated by (7). $\mathrm{n}=2$ and $\mathrm{P}=0.9$ 


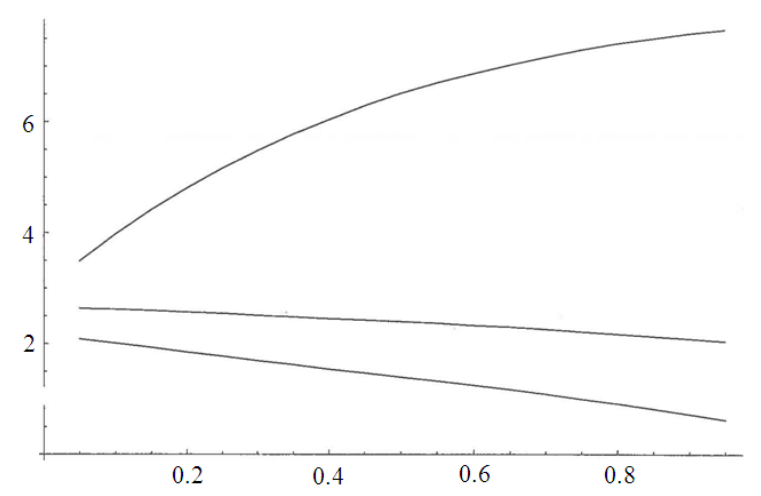

Fig. 5: The equilibrium population $x^{*}$ (map (3)) at instability threshold. Upper curve corresponds to $\mu 2=0.9$, middle curve $\mu 2=0.5$ and bottom curve $\mu 2=0.1$. The stable region is below the curves

Ecological implications: We start by considering density dependent survivals. The asymptotic argument referred to in the beginning of Example 1 shows that precocious iteroparous species possess qualitatively the same dynamics independent of number of age classes both with respect to the size of the stability region and nonstationary behaviour. Additionally, we may also argue that precocious iteroparous species on the whole show much of the same dynamics as precocious semelparous species. Moreover, see Example 2, by performing a rigorous analysis of (6) we may conclude that species with delayed semelparous as well as delayed iteroparous life histories exhibit poorer stability properties than species who possess precocious (iteroparous or semelparous) life histories. However, as is shown by Neubert and Caswell (2000), the analysis of the stage-structured model (3) $\left(\mu_{1}=\mu_{1} \exp (-x)\right.$ and all other parameters constant) does only support parts of the findings reported above. Indeed, they conclude that there are no qualitative differences between populations with precocious or delayed life histories with respect to stability. On the other hand, regarding the nonstationary dynamics, pronounced 4-periodic behaviour has been detected by use of (3) in species which possess precocious semelparous life histories and there are also several examples of 4-periodicity in the delayed semelparous case. Therefore, beyond instability threshold there is an excellent agreement between the outcomes of (1) and (3). Hence, these observations seem to imply that it is a fairly general ecological principle that density dependent growth $\left(\mu_{1}\right)$ in the stage-structured model which in the age-structured model corresponds to density dependent year-to-year survival leads to periodic behaviour of low period. In nature one may find several examples of species who possess cyclic behaviour of low period (Stenseth et al.,
1977; Stenseth and Antonsen, 1988; Burkey and Stenseth, 1994). Especially among lemmings, cf. Stenseth and Ims (1993) this is the case. Lemming cycles are not very well understood. Thereforeriod (Stenseth et al., 1977; Stenseth and Antonsen, 1988; Burkey and Stenseth, 1994). Especially among lemmings, cf. Stenseth and Ims (1993) this is the case. Lemming cycles are not very well understood. Therefore, an interesting and challenging task could be to apply some of the density dependent survival models presented here on small rodent populations.

Next, consider precocious iteroparous species under the assumption of density dependent fecundity terms. From Example 3 we found that $\left(\mathrm{x}_{1}{ }^{*}, \mathrm{x}_{2}{ }^{*}\right)$ is stable in case of $x^{*}$ small. Regarding the nonstationary dynamics there were two scenaria, if $0<\mathrm{P}<1 / 2$ an increase of $\mathrm{x}^{*}$ (or $\mathrm{F}$ ) leads to the period doubling route to chaos. If $1 / 2<\mathrm{P} \leq 1$ the result is given through Fig. 4 . Now, following Wikan and Mjolhus (1996) or Wikan (2012) assuming an odd number of age classes $n$ it is possible to show that the value of $x^{*}$ at instability threshold is an increasing function of both $\mathrm{n}$ and $\mathrm{P}$. In particular, if $\mathrm{P}=1$ then $\mathrm{x}^{*}=\mathrm{n}+1$ at threshold. Nonstationary dynamics is introduced when $\lambda=-1$. Hence, for all $0<\mathrm{P}<1$ the dynamics beyond instability threshold is in many respects similar to what we found in the $0<\mathrm{P}<1 / 2$ case when $n=2$. When $n$ is even and small, $\mathrm{x}^{*}$ is not an increasing function of $\mathrm{P}$ at instability but as $n$ becomes larger $(n \geq 8)$ the dynamics becomes similar to what we find when $n$ is odd. These findings clearly suggest that in case of large or moderate $\mathrm{P}$ values an increase of $\mathrm{n}$ acts stabilizing. See also Levin and Goodyear (1980); Vincent and Skowronski (1981) and Bergh and Getz (1988). Delayed iteroparous species may be studied through the map (Wikan, 2012):

$\left(\mathrm{x}_{1}, \ldots, \mathrm{x}_{\mathrm{n}}\right) \rightarrow\left(\mathrm{f}_{\mathrm{i}} \mathrm{x}_{\mathrm{i}}+\cdots+\mathrm{f}_{\mathrm{n}} \mathrm{x}_{\mathrm{n}}, \mathrm{Px}_{1}, \mathrm{Px}_{2}, \ldots, \mathrm{Px}_{\mathrm{n}-1}\right)$

where, $\mathrm{i}=(\mathrm{n}+1) / 2$ in case of $\mathrm{n} \geq 3, \mathrm{n}$ odd and $\mathrm{i}=\mathrm{n} / 2+$ 1 in case of $n \geq 4$ and $n$ even. $f_{i}=F_{i} \exp (-x)$. By comparing the size of the stable parameter region found from (17) with the precocious situation (7) we find that it is always smaller in the delayed case.

Neubert and Caswell (2000) support the findings above by use of (3) ( $f=F \exp (-x)$ and all other parameters constant). In Fig. 5 we show $x^{*}=x^{*}(p)$ in the cases $\mu_{2}=0.9$ (upper curve), $\mu_{2}=0.5$ (middle curve) and $\mu_{2}=0.1$ (bottom curve). The stable region is below the curves. Hence, the combined findings from the age-structured models and the stage-structured model (large $\mu_{2}$ ) makes it natural to propose that it is a fairly general principle that species with precocious iteroparous life histories are more stable than species 
with delayed iteroparous life histories. Moreover, on the whole, iteroparous species have better stability properties than semelparous species. Still assuming density dependent fecundities our analysis of the semelparous maps (13) and (15) shows that the nontrivial fixed point is unstable $(\beta \leq \alpha)$ both in the delayed and precocious case. Instead, the dynamics is on SYC form. From a biologically point of view it is not obvious how such dynamics should be interpreted.

Bulmer (1977) interprets its presence as "competition is more severe between than within age classes". Mjolhus et al. (2005) argue along the following line: Considering (15) recruitment acts as a birth pulse and juveniles have the same weight as older individuals. Moreover, at equilibrium $\mathrm{FP}^{\mathrm{n}-1} \exp (-\mathrm{x})=1$, i.e., the relation concerns the SYC population when it is in a reproductive phase. In other phases $x=x_{0}+[$ pic $]+x_{n}$ is larger. Therefore, small populations inserted into empty age classes will experience a larger value of $x$ in their reproductive phase than the main population which again will lead to a subcritical recruitment. Wikan (2012) where (13) is analysed, we conclude that it is the strength of density dependence which will decide if the two age classes will coexist or not. Coexistence occurs whenever the strength of density dependence in the survival is stronger $(\beta>\alpha)$ than the density dependence in the fecundity. SYC dynamics is the outcome when $\beta \leq \alpha$. Davydova et al. (2003) who perform a thorough analysis of biennials arrive at a similar conclusion. Also, confer Ebenman (1987; 1988) and Roos et al. (1990) and the papers on cicada cycles by Hoppensteadt and Keller (1976); Heliovaara et al. (1994); Yoshimura (1997); Behncke (2000) and Webb (2001).

\section{CONCLUSION}

As we have seen, nonlinear age- and stagestructured population models serve as excellent tools in order to study the dynamical outcomes of ecological populations who possess different life histories ranging from biennials to species who may live for many years. Depending on parameter values and location on density dependent elements the dynamics may vary from stable fixed point to chaotic behaviour of stunning complexity. Hence, the models at hand which are easily understandable also for readers outside the biological and ecological communities provide excellent examples of nonlinear phenomena. Although the models rest on different prerequisites we find on several occasions that they qualitatively generate much of the same dynamics. This enables us to suggest important general principles with respect to stability and nonstationary behaviour of ecological populations.

\section{ACKNOWLEDGEMENT}

I thank Einar Mjolhus for valuable suggestions.

\section{REFERENCES}

Behncke, H., 2000. Periodical cicadas. J. Math. Biol., 40: 413-431. DOI: $10.1007 / \mathrm{s} 002850000024$

Beland, P., 1974. On predicting the yield from brook trout populations. Trans. Amer. Fish Soc., 103: 353-355. DOI: $\quad 10.1577 / 1548-$ 8659(1974) $103<353$ :OPTYFB > 2.0.CO;2

Bergh, M.O. and W.M. Getz, 1988. Stability of discrete age-structured and aggregated delay-difference population models. J. Math. Biol., 26: 551-581. DOI: $10.1007 / \mathrm{BF} 00276060$

Bernardelli, H., 1941. Population waves. J. Burma Res. Soc., 31: 1-18.

Boling, R.J. Jr., 1973. Towards state-space models for biological populations. J. Theor. Biol., 40: 485506. DOI: 10.1016/0022-5193(73)90006-4

Botsford, L.W., 1986. Population dynamics of the Dungeness crab (Cancer magister). Can. Spec. Publ. Fish. Aquat. Sci., 92: 140-153.

Botsford, L.W., 1992. Further analysis of Clark's delayed recruitment model. Bull. Math. Biol., 54: 275-293. DOI: 10.1016/S0092-8240(05)80027-8

Bulmer, M.G., 1977. Periodical insects. Am. Nat., 111: 1099-1117. DOI: $10.1086 / 283240$

Burkey, T.V. and N.C. Stenseth, 1994. Population dynamics of territorial species in seasonal and patchy environments. Oikos, 69: 47-53. DOI: $10.2307 / 3545282$

Campbell, A. and M.D. Eagles, 1983. Size at maturity and fecundity of rock crabs, Cancer irroratus, from the Bay of Fundy and southwestern Nova Scotia. Fishery Bull., 81: 357-362.

Caswell, H., 2001. Matrix Population Models: Construction, Analysis and Interpretation. 2nd Edn., Sinauer Associates, Sunderland, Massachusetts, ISBN-10: 0878930965, pp: 722.

Clark, C.W., 1976. A delayed recruitment model of population dynamics with an application to baleen whale population. J. Math. Biol., 3: 381-391. DOI: 10.1007/BF00275067

Cooke, D. and J.A. Leon, 1976. Stability of population growth determined by $2 \times 2$ Leslie matrix with density dependent elements. Biometrics, 32: 435442. DOI: $10.2307 / 2529512$

Costantino, R.F., R.A. Desharnais, J.M. Cushing and B. Dennis, 1997. Chaotic dynamics in an insect population. Science, 275: 389-391. DOI: 10.1126/science.275.5298.389 
Crowe, K.M., 1994. A nonlinear ergodic theorem for discrete systems. J. Math. Biol., 32: 179-191. DOI: 10.1007/BF00163877

Cull, P. and A. Vogt, 1973. Mathematical analysis of the asymptotic behaviour of the Leslie population matrix model. Bull. Math. Biol., 35: 645-661. DOI: 10.1007/BF02458368

Cull, P. and A. Vogt, 1974. The periodic limit for the Leslie model. Math. Biosciences, 21: 39-54. DOI: 10.1016/0025-5564(74)90103-5

Cushing, J.M., 1988. Nonlinear matrix models and population dynamics. Nat. Resour. Model, 2: 539580.

Cushing, J.M., 1989. A strong ergodic theorem for some nonlinear matrix models for the dynamics of structured populations. Nat. Resour. Model, 3: 331356.

Cushing, J.M., 1998. An Introduction to Structured Population Dynamics. 1st Edn., SIAM, Philadelphia, Pennsylvania, USA., ISBN-10: 0898714176, pp: 193.

Cushing, J.M., 2006. Nonlinear semelparous Leslie models. Math. Biosci. Eng., 3: 17-36. PMID: 20361805

Cushing. J.M., B. Dennis and R.F. Costantino, 1996. An interdisciplinary approach to understanding nonlinear ecological dynamics. Ecol. Model, 92: 111-119. DOI: 10.1016/0304-3800(95)00170-0

Darwin, J.H. and R.M. Williams, 1964. The effect of time of hunting on the size of a rabbit population. New Zealand J. Sci., 7: 341-352.

Davydova, N.V., O. Diekmann and S.A.V. Gils, 2003. Year class coexistence or competitive exclusion for strict biennials? J. Math. Biol., 46: 95-131. DOI: 10.1007/s00285-002-0167-5

Dennis, B., R.A. Desharnais, J.M. Cushing and R.F. Costantino, 1997. Transition in population dynamics: Equilibria to periodic cycles to aperiodic cycles. J. Anim. Ecol., 6b: 704-729. DOI: $10.2307 / 5923$

Desharnais, R.A. and L. Liu, 1987. Stable demographic limit cycles in laboratory populations of Tribolium Castaneum. J. Anim. Ecol., 56: 885-906. DOI: $10.2307 / 4955$

Devaney, R.L., 2003. An Introduction to Chaotic Dynamical Systems. 2nd Edn., Westview Press, Boulder, Colo., ISBN-10: 0813340853, pp: 335.

Ebenman, B., 1987. Niche differences between age classes and interspecific competition in agestructured population. J. Theor. Biol., 124: 25-33. DOI: $10.1016 / \mathrm{S} 0022-5193(87) 80249-7$
Ebenman, B., 1988. Competition between age classes and population dynamics. J. Theor. Biol., 131: 389400. DOI: $10.1016 / \mathrm{S} 0022-5193(88) 80036-5$

Feigenbaum, M.J., 1978. Quantitative universality for a class of nonlinear transformations. J. Stat. Phys., 19: 25-52. DOI: 10.1007/BF01020332

Getz, W., 1996. A hypothesis regarding the abruptness of density dependence and growth rate of populations. Ecology, 77: 2014-2026. DOI: $10.2307 / 2265697$

Govaerts, W. and R.K. Ghaziani, 2006. Numerical bifurcation analysis of a nonlinear stage structured cannibalism population model. J. Diff. Eq. Appli., 12: 1069-1085. DOI: 10.1080/10236190600986560

Guckenheimer, J. and P. Holmes, 1983. Nonlinear Oscillations, Dynamical Systems and Bifurcations of Vector Fields. 1st Edn., Springer Verlag, New York, ISBN-10: 0387908196, pp: 453.

Guckenheimer, J., G.F. Oster and A. Ipaktchi, 1977. The dynamics of density dependent population models. J. Math. Biol. 4: 101-147. DOI: 10.1007/BF00275980

Gurney, W.S.C., E. McCauley, R.M. Nisbet and W.W. Murdoch, 1990. The physiological ecology of Daphnia, a dynamic model of growth and reproduction. Ecology, 71: 716-732. DOI: $10.2307 / 1940325$

Gyllenberg, M., G. Soderbacka and S. Ericsson, 1993. Does migration stabilize local population dynamics? Analysis of a discrete metapopulation model. Math Biosci., 118: 25-49. DOI: 10.1016/0025-5564(93)90032-6

Hallam, T.G. and S.A. Levin, 1986. Mathematical Ecology: An Introduction. 1st Edn., SpringerVerlag, Berlin, Heidelberg, ISBN-10: 0387136312, pp: 457.

Hallam, T.G., R.R. Lassiter, J. Li and L.A. Suarez, 1990. Modelling individuals employing an integrated energy response. Ecology, 71: 938-954. DOI: $10.2307 / 1937364$

Hastings, A. and L. Botsford, 1999. Equivalence in yield from marine reserves and traditional fishery management. Science, 284: 1537-1538. DOI: 10.1126/science.284.5419.1537

Hastings, A., 1984. Age dependent predation is not a simple process. II. Wolves, Ungulates and a discrete time model for predation on juveniles with a stabilizing tail. Theor. Pop. Biol., 26: 271-282. DOI: 10.1016/0040-5809(84)90033-9

Heliovaara, K., R. Vaisanen and C. Simon, 1994. Evolutionary ecology of periodical insects. Tree, 9: 475-480. DOI: 10.1016/0169-5347(94)90312-3 
Higgins, K., A. Hastings and L.W. Botsford, 1997. Density dependence and age structure: Nonlinear dynamics and population behavior. Am. Nat., 149: 247-269. DOI: 10.1086/285989

Hoppensteadt, F.C. and J.B. Keller, 1976. Synchronization of periodical cicada emergences. Science, 194: 335-337. DOI: 10.1126/science.987617

Jost, J., 2005. Dynamical Systems: Examples of Complex Behaviour. 1st Edn., Springer Science and Business, Berlin, New York, ISBN-10: 3540229086, pp: 189.

Klinkhamer, P.G.L., T.J.D. Jong and E. Meelis, 1987a. Delay of flowering in the 'biennial' Cirsium vulgare: Size effects and devernalization. Oikos, 49: 303-308. DOI: 10.2307/3565765

Klinkhamer, P.G.L., T.J.D. Jong and E. Meelis, $1987 \mathrm{~b}$. Life-history variation and the control of flowering in short-lived monocarps. Oikos, 49: 309-314. DOI: $10.2307 / 3565766$

Kon, R., 2005. Nonexistence of synchronous orbits and class coexistence in matrix population models. SIAM J. Appli. Math., 66: 616-626. DOI: 10.1137/05062353X

Kon, R., Y. Saito and Y. Takeuchi, 2004. Permanence of single species stage-structured models. J. Math. Biol., 48: 515-528. DOI: 10.1007/s00285-0030239-1

Kot, M. and W.M. Schaffer, 1986. Discrete-time growth dispersal model. Math. Biosci., 80: 109136. DOI: 10.1016/0025-5564(86)90069-6

Kuznetsov, Y.A. and H.G.E. Meijer, 2005. Numerical normal forms for Codim 2 bifurcations of fixed points with at most two critical eigenvalues. SIAM J. Sci. Comput., 26: 1932-1954.

Kuznetsov, Y.A., 2004. Elements of Applied Bifurcation Theory. 3rd Edn., Springer Verlag, New York, ISBN-10: 0387219064, pp: 631.

Leslie, P.H., 1945. On the use of matrices in certain population mathematics. Biometrika, 33: 183-212. DOI: $10.1093 /$ biomet/33.3.183

Leslie, P.H., 1948. Some further notes on the use of matrices in population mathematics. Biometrika, 35: 213-245.

Levin, S. and C.P. Goodyear, 1980. Analysis of an agestructured fishery model. J. Math. Biol., 9: 245274. DOI: $10.1007 / \mathrm{BF} 00276028$

Lewis, E.G., 1942. On the generation and growth of a population. Sankhya: Ind. J. Stat., 6: 93-96.

Li, T.Y. and J.A. Yorke, 1975. Period three implies chaos. Amer. Math. Monthly, 82: 985-992. DOI: $10.2307 / 2318254$
Longstaff, B.C., 1977. The dynamics of collembolan populations: A matrix model of single species population growth. Can. J. Zool., 55: 314-324. DOI: $10.1139 / \mathrm{z} 77-043$

Lutscher, F. and M.A. Lewis, 2004. Spatially-explicit matrix model. A mathematical analysis of stagestructured integrodifference equations. J. Math. Biol., 48: 293-324. DOI: 10.1007/s00285-0030234-6

May, R.M. and G.F. Oster, 1976. Bifurcations and dynamical complexity in simple ecological models. Am. Nat., 110: 573-599. DOI: 10.1086/283092

May, R.M., 1976. Simple mathematical models with very complicated dynamics. Nature, 261: 459-467. DOI: 10.1038/261459a0

Metz, J.A.J. and O. Diekmann, 1986. The Dynamics of Physiologically Structured Populations. 1st Edn., Springer-Verlag, Berlin, New York, ISBN-10: 0387167862, pp: 511.

Mjolhus, E., A. Wikan and T. Solberg, 2005. On synchronization in semelparous populations. J. Math. Biol., 50: 1-21. DOI: 10.1007/s00285-0040275-5

Murray, J.D., 2003. Mathematical Biology: Spatial Models and Biomedical Applications. 3rd Edn., Springer-Verlag, Berlin, New York, ISBN-10: 0387952284 , pp: 811.

Neubert, M., M. Kot and M.A. Lewis, 1995. Dispersal and pattern formation in a discrete-time predatorprey model. Theor. Popul. Biol., 48: 7-43. DOI: 10.1006/tpbi.1995.1020

Neubert, M.G. and H. Caswell, 2000. Densitydependent vital rates and their population dynamic consequences. J. Math. Biol., 41: 103-121. DOI: $10.1007 / \mathrm{s} 002850070001$

Nisbet, R.M. and L.C. Onyiah, 1994. Population dynamic consequences of competition within and between age classes. J. Math. Biol., 32: 329-344. DOI: $10.1007 /$ BF00160164

Patten, B.C., 1976. Systems Analysis and Simulation in Ecology. 1st Edn., Academic Press, New York, ISBN-10: 0125472048.

Pennycuick, L., 1969. A computer model of the Oxford great tit population. J. Theor. Biol., 22: 381-400. DOI: 10.1016/0022-5193(69)90011-3

Ricker, W.E., 1954. Stock and recruitment. J. Fish. Res. Board Can., 11: 559-623. DOI: 10.1139/f54-039

Roos, A.M.D., J.A.J. Metz, E. Evers and A. Leipoldt, 1990. A size dependent predator-prey interaction: Who pursues whom? J. Math. Biol., 28: 609-643. DOI: $10.1007 / \mathrm{BF} 00160229$ 
Sarkovskii, A.N., 1964. Coexistence of cycles of a continuous map of a line into itself. Ukr. Math. Z., 16: 61-71.

Silva, J.A. and T.G. Hallam, 1993. Effects of delay, truncation and density dependence in reproduction schedules on stability of nonlinear Leslie matrix models. J. Math. Biol., 31: 367-395. DOI: 10.1007/BF00163922

Singer, D., 1978. Stable orbits and bifurcations of maps of the interval. SIAM J. Applied Math., 35: 260267. DOI: $10.1137 / 0135020$

Stenseth, N.C. and A. Ims, 1993. The Biology of Lemmings. 1st Edn., Academic Press, London, Sydney, ISBN-10: 0126660204, pp: 683.

Stenseth, N.C. and B.J. Antonsen, 1988. Density dependence and the microtine cycle II. A discrete time model for a synchronously reproducing population. Oikos, 52: 207-211. DOI: $10.2307 / 3565249$

Stenseth, N.C., L. Hansson, A. Myllymaki and J. Katila, 1977. General models for the population dynamics of the field vole microtus agretis in central Scandinavia. Oikos, 29: 616-642. DOI: $10.2307 / 3543600$

Thunberg, H., 2001. Periodicity versus chaos in onedimensional dynamics. SIAM Rev., 43: 3-30. DOI: 10.1137/S0036144500376649

Tuljapurkar, S., C. Boe and K.W. Wachter, 1994. Nonlinear feedback dynamics in fisheries: Analysis of the Deriso-Schnute model. Can. J. Fish Aquat. Sci., 51: 1462-1473. DOI: 10.1139/f94-146

Ueta, T., G. Chen, T. Yoshinaga and H. Kawakami, 1999. A numerical algorithm for computing Neimark-Sacker bifurcation parameters. Circ. Syst., 5: 503-506. DOI: 10.1109/ISCAS.1999.777619

Ugarcovici, I. and H. Weiss, 2004. Chaotic dynamics of a nonlinear density dependent population model. Nonlinearity, 17: 1689-1711. DOI: 10.1088/09517715/17/5/007
Vincent, T.L. and J.M. Skowronski, 1981. Renewable Resource Management. 1st Edn., Springer-Verlag, Heidelberg, ISBN-10: 3540105662, pp: 236.

Wan, Y.H., 1978. Computation of the stability condition for the Hopf bifurcation of difeomorphisms on $\mathrm{R}^{2}$. SIAM J. Applied Math., 34: 167-175. DOI: 10.1137/0134013

Webb, G.F., 2001. The prime number periodical cicada problem. Discrete Cont. Dyn. Sys-Ser. B, 1: 387-399.

Wikan, A. and E. Mjolhus, 1995. Periodicity of 4 in age-structured population models with density dependence. J. Theor. Biol., 173: 109-119. DOI: 10.1006/jtbi.1995.0048

Wikan, A. and E. Mjolhus, 1996. Overcompensatory recruitment and generation delay in discrete agestructured population models. J. Math. Biol., 35: 195-239. DOI: $10.1007 / \mathrm{s} 002850050050$

Wikan, A., 1997. Dynamic consequences of reproductive delay in Leslie matrix models with nonlinear survival probabilities. Math. Biosci., 146: 37-62. DOI: 10.1016/S0025-5564(97)00074-6

Wikan, A., 1998. Four-periodicity in Leslie matrix models with density dependent survival probabilities. Theor. Popul. Biol., 53: 85-97. DOI: 10.1006/tpbi.1997.1344

Wikan, A., 2012. Age or stage structure? A comparison of dynamic outcomes from discrete age- and stagestructured population models. Bull. Math. Biol., DOI: $10.1007 / \mathrm{s} 11538-012-9715-3$

Yoshimura, J., 1997. The evolutionary origins of periodical cicadas during ice ages. Am. Nat., 149: 112-124. DOI: $10.1086 / 285981$ 